\title{
Nonmetric scaling of line lengths using latencies, similarity, and same-different judgments ${ }^{1}$
}

\section{FORREST W. YOUNG ${ }^{2}$ UNIVERSITY OF NORTH CAROLINA}

\begin{abstract}
An experiment investigating the relation between various ways of deriving psychophysical functions for line length is reported. A two-phase computer-controlled experiment in which the stimuli were pairs of lines was performed. In the first phase, the pair was presented for several seconds and the $S$ was required to make a category judgment of the similarity of the lines. In the second phase, the pair was presented for a very brief period of time and the $S$ decided whether the lines were the same or of different lengths. Three sets of data were analyzed: (1) the similarity judgments, (2) the proportion of correct different judgments, and (3) the latency of the correct different judgments. An individual differences analysis indicated that there were individual differences in the similarity and latency data. This analysis was not applicable to the proportion data. Nonmetric scaling revealed that the psychophysical functions based on all three sets of data were essentially identical.
\end{abstract}

Shepard, in his paper on the analysis of proximities (1962), suggested that many empirical procedures have one thing in common: They start with a fixed set of objects and determine, for all pairs of the objects, some number which reflects how closely the two objects are related psychologically. In psychophysics the numbers reflecting the relation of the pairs of stimuli are called similarities.

The purpose of the research reported here is to gather and analyze various kinds of similarities data about one set of stimuli: lines varying in length. Recently Markley, Ayers, and Rule (1969) gathered two kinds of similarities data about line length. One set of data was from a similarities-estimation procedure in which the $S s$ were asked to respond with a number from 0 to 100 . The other set of data was collected using a magnitude-estimation procedure in which the Ss were presented with an initial pair (to which the $E$ assigned a value of 10 ) and then judged the ratio of the similarity of subsequent pairs to the initial pair. The results of the study indicated that nonmetric analysis produced equivalent sets of scale values for the two sets of data and that these scale values were logarithmically related to the physical length of the lines.

The Markley et al study indicates that magnitude-estimation judgments can serve as similarity measures just as easily as category judgments of similarity. This is gratifying, although not surprising, since both procedures involve some form of numerical estimation. For two sets of numbers to serve equally well as similarity measures, it is only necessary (but not sufficient) for the two sets of numbers to be highly correlated. More specifically, with a nonmetric analysis. it is only necessary for the two sets of numbers to have a high ordinal correlation. We should be gratified, then, that the similarity-estimation procedure and the magnitude-estimation procedure yield equivalent scale values, since this implies that the raw data from one procedure is ordinally correlated with that from the other procedure. That is, these two numerical estimation procedures are at least ordinally equivalent.

The purpose of the present research is to investigate whether data other than numerical estimates of similarity can serve as similarity indices. Specifically, this research investigates whether same-different judgments and the latency of same-different judgments can serve as similarity measures. Brown and Andrews (1968) have investigated the use of latencies as a similarity measure in a discrimination experiment. This procedure did not permit them to obtain other similarity measures for comparison with the latency measures, although they did compare their results with those of Behrman and Brown (1968). The procedure used here let us obtain two similarity estimates from each. In order to provide an additional means of evaluating the adequacy of the same-different procedure, the research also involved gathering similarity-estimation data. By comparing the psychological functions and goodness-of-fit indices from the same-different paradigm with the corresponding information from the similarity-estimation paradigm, we are able to evaluate the relative desirability of each paradigm.

\section{METHOD}

Subjects

The Ss were 15 students enrolled in an introductory course in psychology. Participation in the experiment met a course requirement.

\section{Stimuli}

The stimuli were 14 horizontal lines with display lengths varying from 10.75 to $14.044 \mathrm{~cm}$ in equal increments of $.305 \mathrm{~cm}$.

\section{Apparatus}

The experiment was run at the computer-controlled experimentation facility at the L. L. Thurstone Psychometric Laboratory. The experiment was administered by a Digital Equipment Corporation PDP-8 computer using the system for administering visual experiments (SAVE). The facility, computer, and software system have been discussed by Jones, Johnson, and Young (1970).

The stimuli were presented via a visual display device built by Computer Control, Inc. (Model CC-30). The device uses an ordinary 12-in. Sony television receiver to display an 85 by 108 grid of points. The entire grid is $16.5 \mathrm{~cm}$ long $\times 13.4 \mathrm{~cm}$ high. Any combination of all 9,180 points may be displayed. The points are $.1527 \mathrm{~cm}$ apart horizontally and $.1689 \mathrm{~cm}$ apart vertically.

Each of the stimuli consisted of one row of points. For a pair of lines, the top line was presented on the 21 st row, the bottom on the 64th. This spacing placed the two lines equidistant from the top and bottom edges of the display, with a separation of $7.26 \mathrm{~cm}$. Each line was horizontally centered on the middle of the row.

The instructions for the experiment were presented via the display device using the alphameric mode.

The responses were made on a standard Model ASR-33 Teletype. At the end of each trial the computer punched, on paper tape, information about the trial, including $S$ 's response latency. The $S$ was located in a sound-attenuated $8 \times 8 \mathrm{ft}$ experimental 
Fig. 1. Individual differences in similarities judgments. Idiosyncratic $S$ represented by $\Delta$, main subgroup by $\nabla$, Subgroup 2 by 0 , and Subgroup 3 by $\square$. Solid shapes represent idealized individuals used to generate similarities.

cubicle. Also located in the cubicle were the display unit and the Teletype. The remaining equipment, including the computer and paper tape punch, was located in a separate room.

\section{Procedure}

The $S$ was seated at the Teletype directly in front of and $4 \mathrm{ft}$ from the display unit. The $S$ was told that the instructions for the experiment would be presented on the display unit.

There were five phases to the experiment. All $\mathrm{Ss}$ received the same first phase. The purpose of this initial phase was to instruct the $S$ in the use of the equipment and to familiarize him with the purposes of the experiment. The $S$ was shown the entire set of 14 stimuli one at a time. Each stimulus was displayed for $2 \mathrm{sec}$ with an interstimulus interval of $1 \mathrm{sec}$. The stimuli were shown in one random order to all Ss. The Ss were instructed to look at each stimulus carefully, to try to remember particularly what the longest and shortest stimuli were like.

At the completion of the initial familiarization phase, the experiment proper began. There were two different experimental conditions, a similarity (SIM) condition and a same-different (S-D) condition. Each $S$ participated in the SIM condition once and in the S-D condition three times. Due to equipment difficulties, data for two Ss were lost for one presentation of the S-D condition. There were, then, 15 sets of SIM data and 43 sets of S-D data. The order of the conditions was randomized across Ss, with the SIM condition appearing first four times, second four times, third three times, and last four times. The order was randomized to reduce confounding due to fatigue and boredom. The $S$ received a short break between each phase.

Both SIM and S-D conditions involved stimulus pairs that consisted of all 105 combinations of the 14 lines. Pairs were formed by using a Ross ordering (Ross, 1938) for 15 stimuli. Every time a stimulus would have been paired with Number 15 (which did not exist), it was paired with itself instead. In this manner, the top-bottom position of each individual line was counterbalanced, with every line being presented before a given line was presented again. The Ross order eliminates order effects between individual stimuli. The same order was used for all Ss and in all

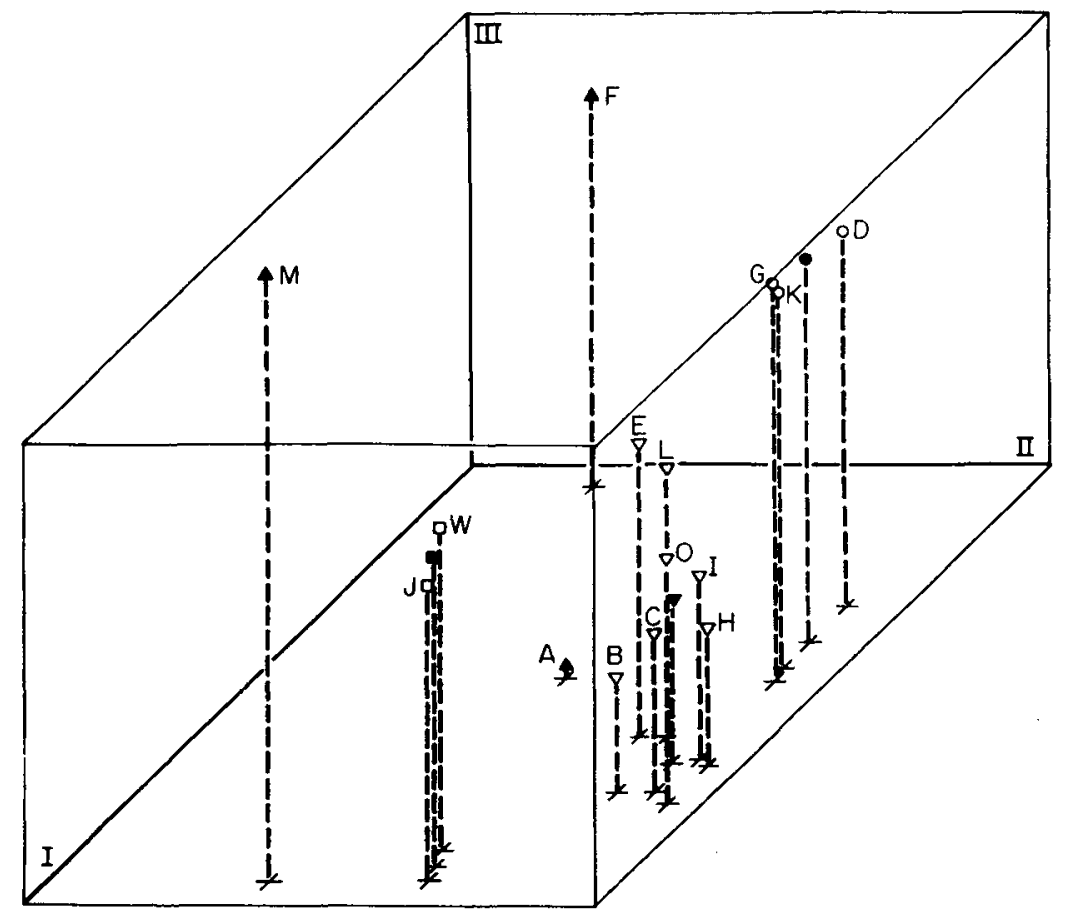

conditions. Through a clerical error, one pair was presented twice and one pair was omitted.

In the SIM condition the Ss were instructed (by the display) to decide how different the two lines in each pair were from each other and to respond by typing a number from 1 to 9 . They were told that there would be identical pairs and that those should be assigned the response 1 . They were also told to reserve 9 for the most different pair. The Ss were urged to respond quickly but were given as much time to respond as they desired. Immediately upon responding, the stimulus disappeared and a new stimulus appeared 1 sec later. Following the instructions, the $S$ was shown all 105 pairs of stimuli.

In the S-D condition, the $S$ was shown each stimulus pair for $.16 \mathrm{sec}$. He then had an additional $4.84 \mathrm{sec}$ to type an " $S$ " or a " $D$," depending on whether he thought the two lines were the same or of different lengths. The $S$ was simply instructed to respond during the $5-\mathrm{sec}$ period. He was unaware that the latency of his response was being recorded and was not instructed to respond quickly. Following the $5-\mathrm{sec}$ response period was a $1-\mathrm{sec}$ intertrial interval. The entire experiment lasted about $1 \mathrm{~h}$.

\section{RESULTS AND DISCUSSION}

\section{Similarity Judgments:}

\section{Individual Differences}

The raw similarity judgments were submitted to a Tucker and Messick points-of-view analysis (Tucker \& Messick,
1963; Young \& Pennell, 1967) to investigate the possibility of individual differences. The analysis was performed on the intersubject correlations and is similar to an inverse factor analysis (see Ross, 1966; Cliff, 1968). On the basis of the size of the eigenvalues it was decided that the individual-differences space was three-dimensional. A plot of the space is presented in Fig. 1. The axes are numbered according to the percentage variance accounted for: Axis 1 accounts for the most, etc.

The $S$ space seemed to contain three idiosyncratic Ss, A, F, and M. S A used only Categories 1,2 , and 3 . It was not possible to identify the idiosyncracy of Ss $F$ and $M$. In addition, the individual-differences space appeared to contain three subgroupings of Ss: one for Ss B, C, E, I, L, H, and O (referred to as the main subgroup, since almost half the $S$ s are included in it), one for $S s D, G$, and $K$ (referred to as Subgroup 2), and one for Ss $J$ and $W$ (Subgroup 3). Accordingly, six idealized individuals were constructed: three corresponding to each of the three idiosyncratic Ss and three corresponding to the approximate centroids of each of the subgroups. The locations of the six idealized individuals are represented by the darkened shapes in Fig. 1.

Six sets of idealized judgments were generated as suggested by Cliff (1968). Each set of judgments was submitted to the TORSCA nonmetric scaling program (Young \& Torgerson, 1967). These analyses, and all the other nonmetric 


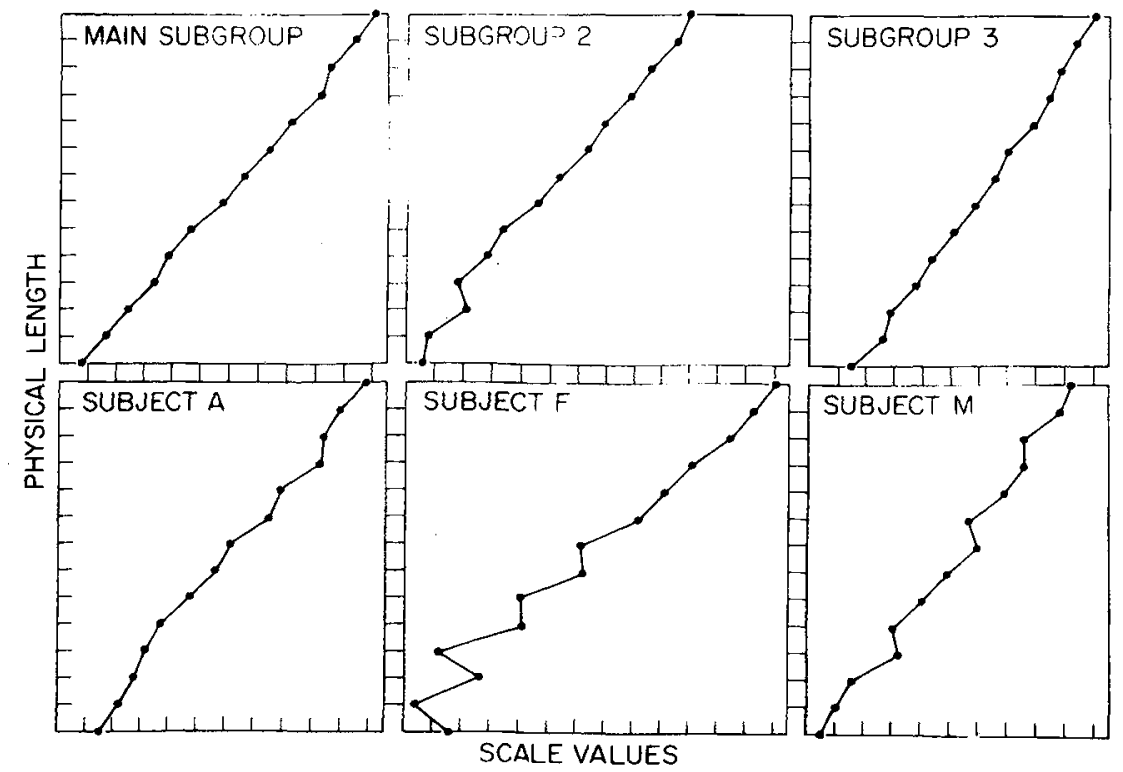

scaling analyses reported in this paper, were performed in Euclidian spaces of both one and two dimensions. Specifically, the raw data was first analyzed according to the metric multidimensional scaling equations presented by Torgerson (1960). The largest two dimensions obtained by this analysis served as the initial configuration for the two-dimensional nonmetric scaling. The monotonically transformed raw data resulting from the two-dimensional analysis then served as the basis for the one-dimensional analysis. Specifically, the Torgerson metric equations were applied to the monotonically transformed raw data, and the largest dimension obtained by this analysis served as the initial configuration for the one-dimensional nonmetric scaling.

Two sets of scale values (one set one-dimensional, the other two-dimensional) were obtained for each of the six sets of idealized judgments. The one-dimensional set of scale values is presented in Fig. 2 as a function of the physical length of the stimuli. In the remainder of this paper, the function obtained by plotting a one-dimensional set of scale values vs the physical lengths will be referred to as the psychophysical function in a given set of data.

Perhaps the most readily apparent feature of Fig. 2 is that the psychophysical functions derived from the idiosyncratic $S s$ $F$ and $M$ differ markedly from the other psychophysical functions. First of all, the derived scale values are not monotonically related to the physical values. Second, the

Fig. 3. Two-dimensional similarity-judgment spaces for three idealized individuals. goodness of fit of these two solutions to their data, as indicated by Kruskal's stress index (1964) was poor, with $S=.311$ for $\mathrm{SF}$, and $\mathrm{S}=.305$ for $\mathrm{S} \mathrm{M}$. Various Monte Carlo research (Young, 1970; Stenson \& Knoll, 1969) suggests that stress values this large are obtained when random data is analyzed. This may indicate that these two sets of data contain no underlying structure.

The psychophysical function derived from the third idiosyncratic $S$ (S A) is, on the other hand, very similar to the functions derived from the three group points of view. The scale values are monotonically related to the physical lengths, and the goodness of fit, $S=.180$, suggests that this $S$, while behaving differently from the overall group, was not behaving randomly. It is interesting to refer back to Fig. 1, the individual differences space. Here we note that $S s F$ and $M$, whose psychophysical functions are clearly different, are much further from the main cluster of $S$ s than $S A$.

Returning to Fig. 2, we note that the solutions for the three subgroups are all very similar in terms of their psychophysical functions. The stress values of the three subgroups are not the same, however. The stress values for Subgroups 2
Fig. 2. Psychophysical functions derived from the similarity judgments for six idealized individuals.

and 3 are .169 and .185 , respectively, whereas the stress of the main subgroup is considerably better (.089).

By looking at the two-dimensional solutions for the three subgroups, we get a better idea of what the differences may be in the individual judgment processes. The two-dimensional solutions are presented in Fig. 3, where we see that the two $S$ s in Subgroup 3 are apparently viewing the stimuli as a heterogeneous set of stimuli composed of "longs," "mediums," and "shorts." It appears that these two Ss are using two different bases for their judgments: (1) When comparing stimuli within a class (such as within "longs") or, to a lesser extent, between adjacent classes (such as between "longs" and "mediums"), the judgments are based on the physical differences in length; (2) when comparing stimuli between classes (particularly between "longs" and "shorts"), the judgments are simply based on class differences (that is, all the longs are judged to be approximately the same difference from all the shorts). This effect is most pronounced for Subgroup 3 but is also present for Subgroup 2. There is no such effect for the main subgroup whose judgments are most clearly one-dimensional.

\section{Similarity Judgments:}

Psychophysical Function

We now turn to the form of the psychophysical function for the main subgroup. It is clearly linear: Subjective length (as revealed by nonmetric scaling) is a linear function of physical length when the judgments are based on ratings of the difference of pairs of lines. From Fig. 4, which presents the monotonic function (derived by the scaling program) relating judged difference and Euclidian distance for the main subgroup, we can further conclude, for the experimental paradigm under discussion, that the distance between scale values is a linear function of the judged difference of the stimuli. Furthermore, noting the linear

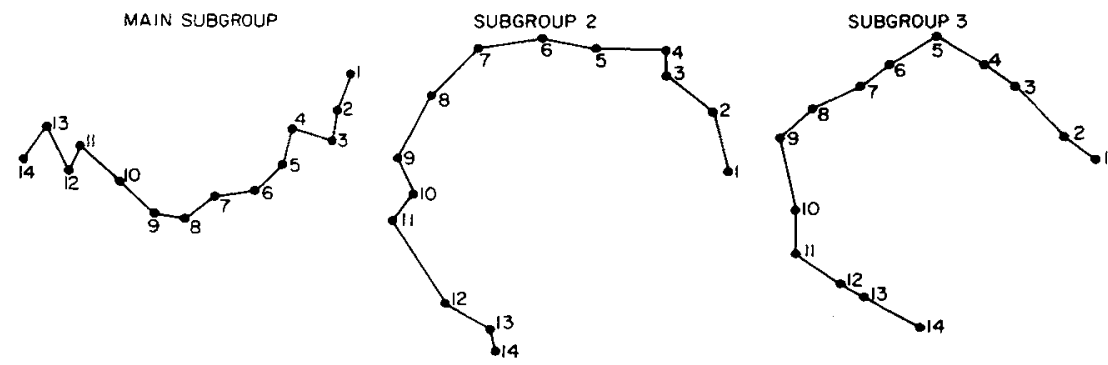



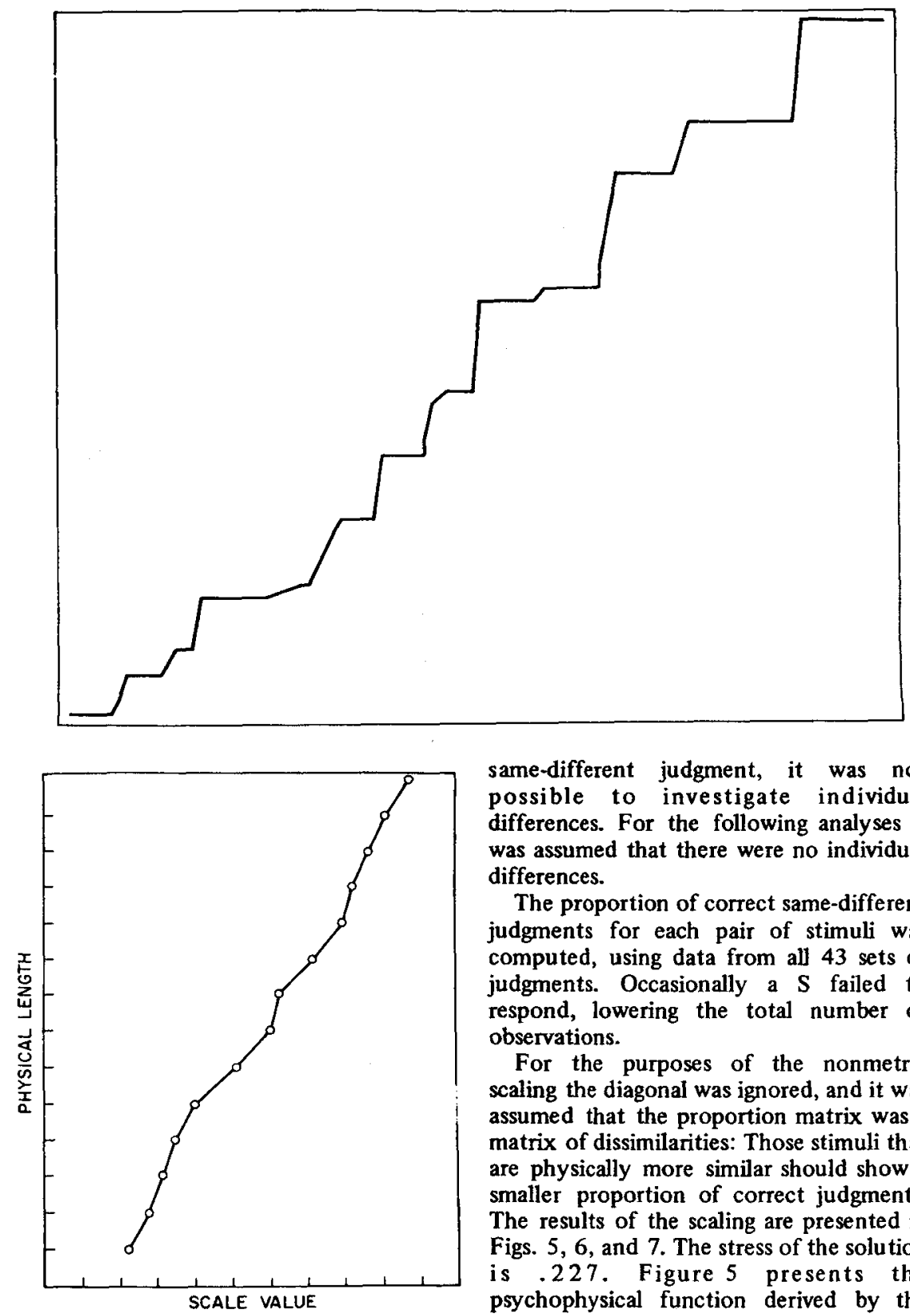

Fig. 5. Psychophysical function derived from the proportion of correct same-different judgments.

psychophysical function, we can see that judged difference between stimuli is linearly related to the physical distance between line lengths. These conclusions are at odds with those presented by Markley et al (1969). The disagreement in findings may be due to the differences in the experimental paradigms.

\section{Same-Different Judgments:}

Psychophysical Function

Because of the nature of the same-different judgment, it was not possible to investigate individual differences. For the following analyses it was assumed that there were no individual differences.

The proportion of correct same-different judgments for each pair of stimuli was computed, using data from all 43 sets of judgments. Occasionally a $S$ failed to respond, lowering the total number of observations.

For the purposes of the nonmetric scaling the diagonal was ignored, and it was assumed that the proportion matrix was a matrix of dissimilarities: Those stimuli that are physically more similar should show a smaller proportion of correct judgments. The results of the scaling are presented in Figs. 5, 6, and 7. The stress of the solution is .227 . Figure 5 presents the psychophysical function derived by the scaling procedure. Again, this is a linear function. The close similarity between this function and the one based on the main subgroup from the similarities analysis (Fig. 2) is emphasized in Fig. 6, where the function relating to the two sets of scale values is presented. Clearly, the two paradigms have permitted us to derive the same psychophysical function. However, the monotonic function (presented in Fig. 7) relating the proportion matrix and the derived distances differs markedly from the one in the similarities analysis. As indicated in Fig. 4, for the similarities data the transformation is very nonlinear. It is interesting that we were able to derive essentially identical scale values from two
Fig. 4. Best monotonic transformation of similarities data for the main subgroup.

sets of data that are not linearly related to each other.

\section{Same-Different Latencies: Individual Differences}

A Tucker and Messick points-of-view analysis was performed on the 43 sets of raw S-D latencies. This analysis revealed that $S \mathrm{~L}$ was idiosyncratic in all three sets of S-D data. It was observed that his mean latency and the variance of his latencies were both larger than those of any of the other Ss. The $S$ space revealed no subgrouping of Ss.

\section{Same-Different Latencies: \\ Psychophysical Function}

The psychophysical function was based on data from all Ss except S $L$.

It would have been possible to obtain the similarities by computing, for each pair of stimuli, the mean of the 40 observed latencies. This was not done, however, because previous experience indicated that the latency of a same-different judgment depends on whether the judgment was right or wrong. Averaging overall judgments confounds two unique judgment processes: one underlying correct judgments and one underlying incorrect judgments. For this reason, the judgments were first separated into those that were correct and those that were incorrect, and then the mean correct and mean incorrect judgment latency was obtained for all pairs of stimuli. This procedure gives two similarity matrices, one of mean latencies for correct judgments and one of mean latencies for incorrect judgments. It should be noted that the number of observations

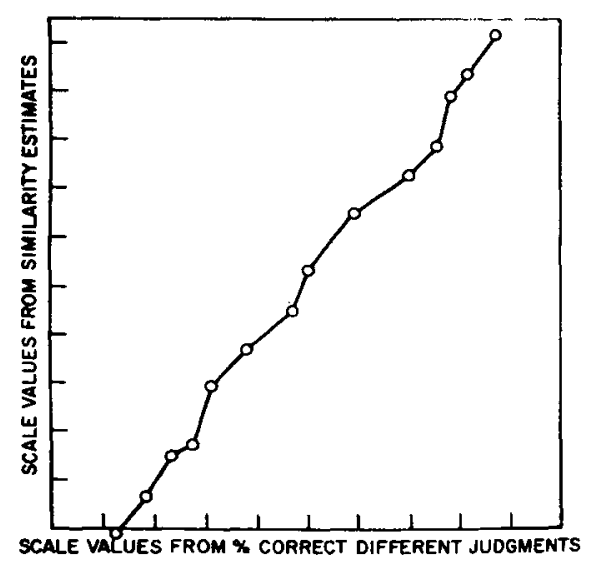

Fig. 6. Relation bewteen psychophysical functions derived from main subgroup similarity judgments and proportion of correct same-different judgments. 
Table I

Mean Latencies for Different Judgments

\begin{tabular}{|c|c|c|c|c|c|c|c|}
\hline \multirow{2}{*}{$\begin{array}{l}\text { Stimulus } \\
\text { Number }\end{array}$} & \multicolumn{7}{|c|}{ Stimulus Number } \\
\hline & 1 & 2 & 3 & 4 & 5 & 6 & 7 \\
\hline 1 & & $1213(38)$ & $1115(24)$ & $1508(17)$ & $1416(8)$ & & \\
\hline 2 & $1205(2)$ & & $1127(37)$ & $1227(26)$ & $1310(17)$ & $1351(6)$ & $1418(5)$ \\
\hline 3 & $1528(16)$ & $1699(3)$ & & $1028(38)$ & $1259(34)$ & $1513(9)$ & $1322(10)$ \\
\hline 4 & $1531(23)$ & $1619(14)$ & $1762(2)$ & & $1244(31)$ & $1210(33)$ & $1180(7)$ \\
\hline 5 & $1407(32)$ & $1386(22)$ & $1754(6)$ & $1694(9)$ & & $1345(28)$ & $1292(31)$ \\
\hline 6 & $1268(39)$ & $1337(34)$ & $1240(31)$ & $1730(7)$ & $1760(12)$ & & $1079(34)$ \\
\hline 7 & $1079(40)$ & $1262(35)$ & $1309(30)$ & $1262(33)$ & $1416(9)$ & $1522(6)$ & \\
\hline 8 & $1029(40)$ & $1114(38)$ & $1294(34)$ & $1258(33)$ & $1368(23)$ & $1520(9)$ & $1390(4)$ \\
\hline 9 & $1148(40)$ & $1140(40)$ & $1164(38)$ & $1156(40)$ & $1284(36)$ & $1362(31)$ & $1644(18)$ \\
\hline 10 & $1004(38)$ & $985(40)$ & $1041(39)$ & $1155(38)$ & $1160(40)$ & $1407(35)$ & $1295(21)$ \\
\hline 11 & $1028(40)$ & $1103(40)$ & $1082(40)$ & $1051(40)$ & $1118(40)$ & $1324(32)$ & $1456(37)$ \\
\hline 12 & $1014(40)$ & $928(40)$ & $1054(40)$ & $1159(38)$ & $1054(40)$ & $1014(40)$ & $1289(30)$ \\
\hline 13 & $946(40)$ & $1063(38)$ & & $1071(40)$ & $1063(39)$ & $1033(40)$ & $1164(37)$ \\
\hline \multirow[t]{2}{*}{14} & $1112(40)$ & $933(40)$ & $1043(40)$ & $949(40)$ & $964(40)$ & $1058(39)$ & $1164(37)$ \\
\hline & 8 & 9 & 10 & 11 & 12 & 13 & 14 \\
\hline 1 & & & $1360(2)$ & & & & \\
\hline 2 & $1084(2)$ & & & & & $1896(1)$ & \\
\hline 3 & $1386(6)$ & $1254(2)$ & $1854(1)$ & & & & \\
\hline 4 & $1484(7)$ & & $2317(2)$ & & $2098(2)$ & & \\
\hline 5 & $1257(17)$ & $1217(4)$ & & & & $928(1)$ & \\
\hline 6 & $1086(31)$ & 1294 (19) & $1814(5)$ & $1585(8)$ & & & \\
\hline 7 & $1279(36)$ & $1183(22)$ & 1204 (19) & $1387(3)$ & $1562(10)$ & $1270(3)$ & $2368(2)$ \\
\hline 8 & & $1515(27)$ & $1385(20)$ & $1291(7)$ & $1434(9)$ & $1275(6)$ & \\
\hline 9 & $2123(12)$ & & $1177(39)$ & $1206(17)$ & $1273(23)$ & $1467(14)$ & \\
\hline 10 & $1433(19)$ & & & $1185(38)$ & $1489(19)$ & $1157(33)$ & $1494(10)$ \\
\hline 11 & $1192(33)$ & $1299(23)$ & $1491(2)$ & & $1376(30)$ & $1524(16)$ & $1630(8)$ \\
\hline 12 & $1327(31)$ & $1454(17)$ & $1443(21)$ & $1303(10)$ & & $1306(27)$ & $1283(31)$ \\
\hline 13 & $1234(34)$ & $1301(26)$ & $1697(7)$ & $1210(24)$ & $1563(13)$ & & $1103(39)$ \\
\hline 14 & $1141(40)$ & $1068(40)$ & $1349(30)$ & $1215(32)$ & $1680(9)$ & $1043(40)$ & \\
\hline
\end{tabular}

Note-Latencies are in milliseconds. Number in parentheses is number of observations. (Empty cell represents no observation.) Upper triangle is for incorrect judgments, lower is for correct judgments. Rows and columns arranged in order according to stimulus length (Row $I$ and Column 1 for shortest stimulus, etc.).

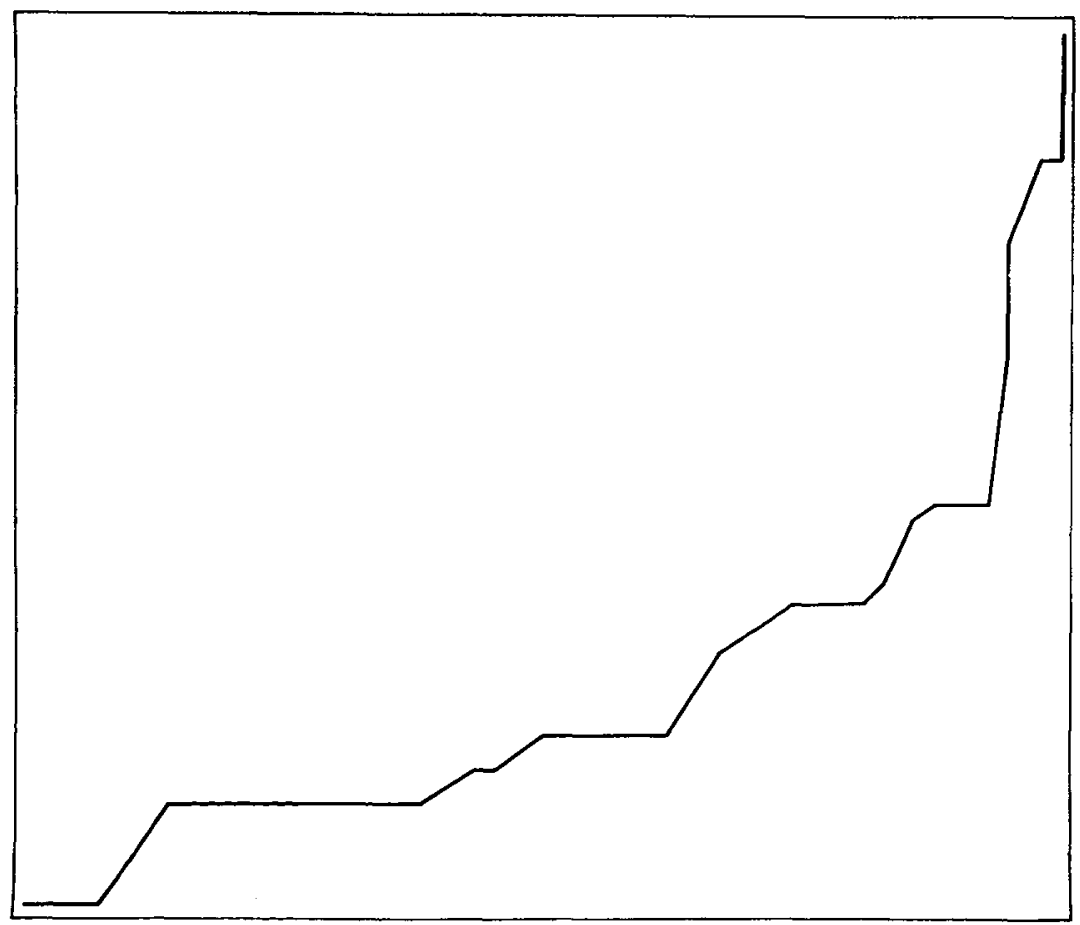

Fig. 7. Best monotonic transformation of proportion of correct same-different judgments.

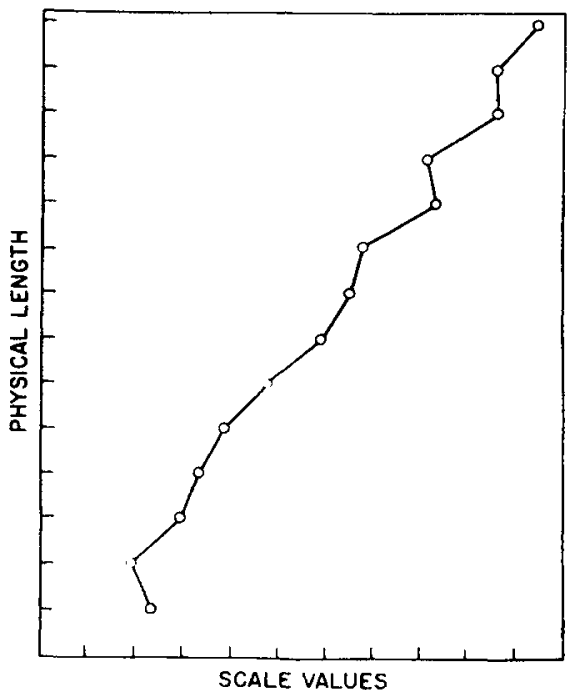

Fig. 8. Psychophysical function derived from the latency of correct same-different judgments.

underlying each mean varies from 0 to 40 . The mean latencies in milliseconds and the number of observations underlying each mean are presented in Table 1. The upper triangle is for incorrect judgments, and the lower for correct. Occasionally the number of incorrect and correct judgments does not total 40 because a $S$ did not respond. Note that there are many cells, especially in the matrix of incorrect-judgment latencies, with no entry. For these cells the frequency of occurrence was zero. The matrix of incorrect-judgment latencies was not analyzed as there was too much missing data.

Finally, note that the latency of judgment for a stimulus paired with itself was not analyzed even though it was available. This explains the fact that there are no diagonal entries in Table 1 . The psychophysical function derived by the nonmetric program from the latencies of correct judgments is presented in Fig. 8. The function, while not as smooth as those derived earlier, generally indicates a linear relationship between physical length and scale values. It should be noted that the stress of this solution is similar to those of the previous solutions, being .203. The monotonic function (derived by the program) relating mean latencies and the derived Euclidian distances is presented in Fig. 9. We can see that a nonlinear transformation was derived. Finally, the relation between the scale values obtained here, and those obtained from the main subgroup of the SIM data is presented in Fig. 10. Here we can see that we have derived essentially the same scale values from the S-D latencies as from the SIM judgments and, by implication (see Fig. 6), 


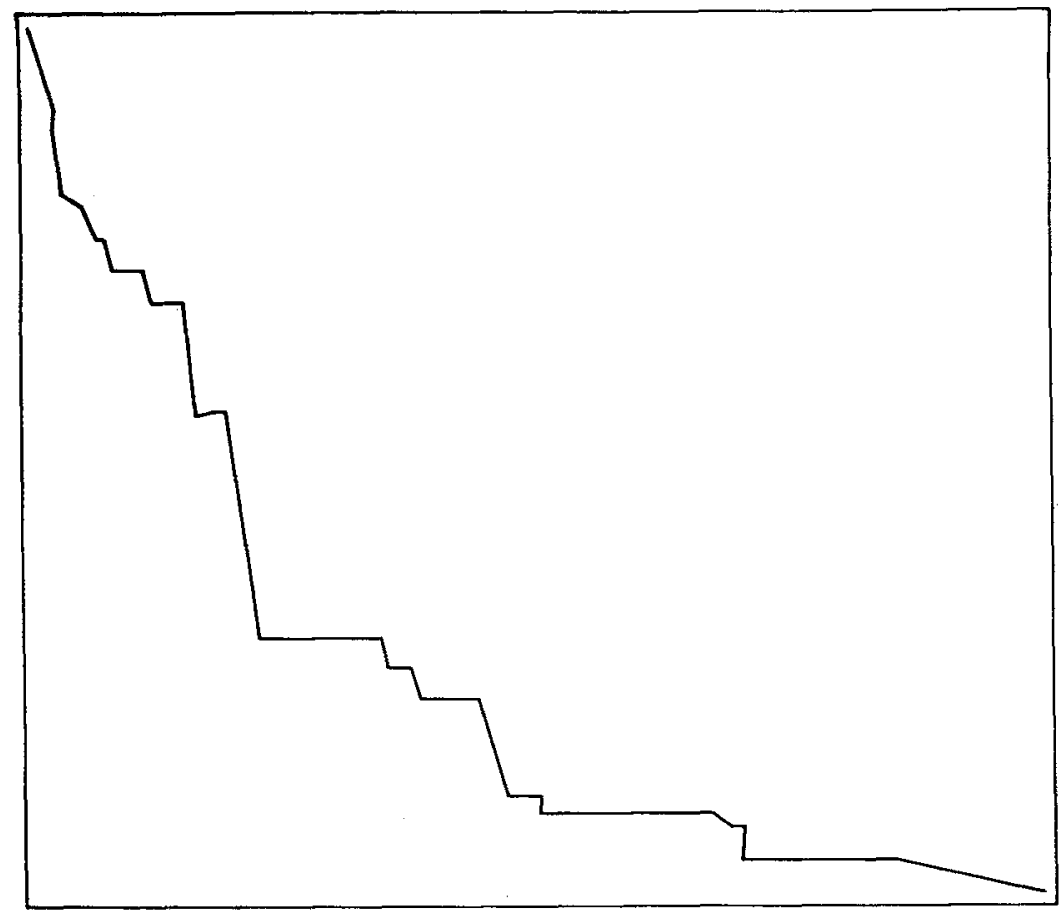

as from the percentage of correct S-D judgments. The only apparent difference between the three sets of scale values is that the one derived from the latencies appears to be less "well behaved." While it is more jagged than the others, it does not appear to differ in any consistent manner, suggesting that the latency data is more prone to error than are the other sets of data.
It is, perhaps, important to emphasize the nonlinear relations between the three sets of raw data. The nonlinear relationship can be seen by looking at the three monotonic functions derived in each analysis (Figs. 4, 7, and 9). Since the scale values are linearly related, the Euclidian distances (vertical axes) are also linearly related. We can, therefore, directly compare the three monotonic

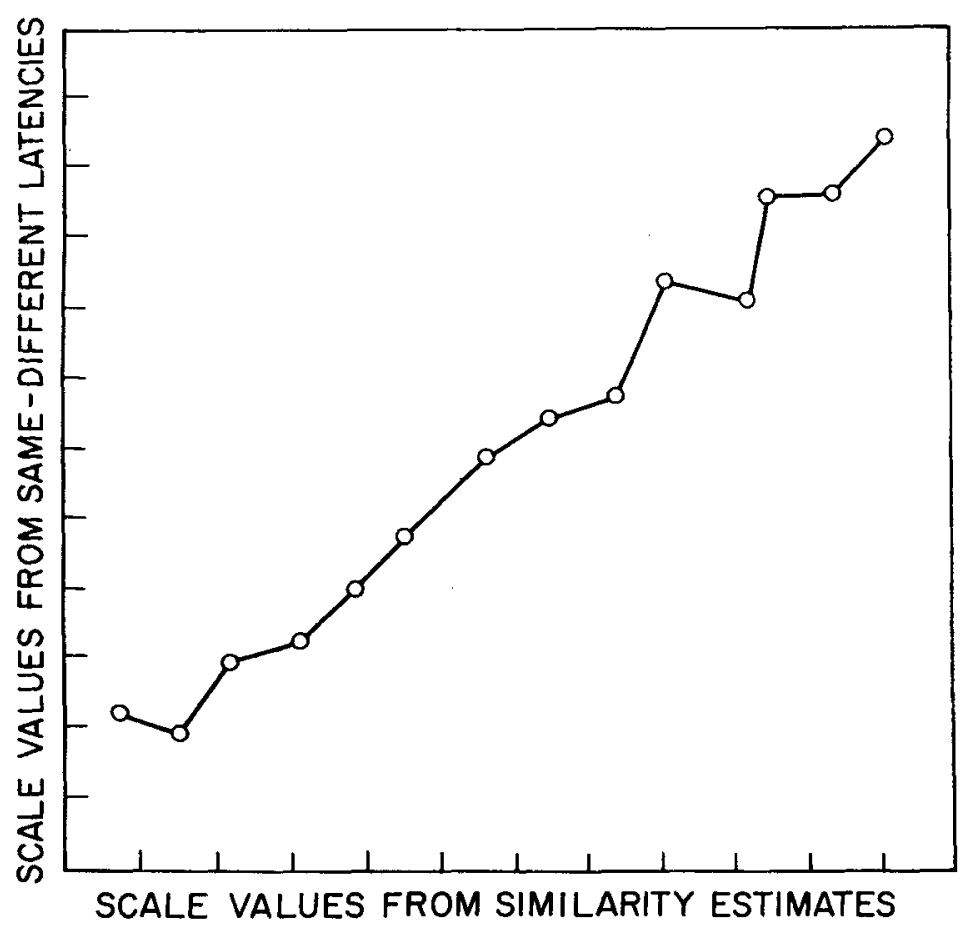

for latency of correct same-different judgments.

transformations, noting that the three sets of data are not related in any direct linear way. It is possible, had we used a metric analysis, that the nonlinearities would have kept us from recovering the same scale values for the three sets of data. While it is true that the three sources of data allow us to derive equivalent sets of scale values, it is not true that all three sources are equally desirable: Some of the methods for gathering data produce data with less Euclidian information (more "noise" in the current context) and some of the methods require more effort on the part of the $S$. The S-D paradigm suffers in both these ways. The stress values for S-D judgments (.227) and latencies (.203) as compared with stress for SIM judgments (.089) indicate that there is more "noise" produced by the S-D paradigm. Furthermore, the S-D paradigm involved three times the number of judgments as did the SIM paradigm. In fact, the S-D experiment was repeated three times precisely because pilot experiments indicated that one presentation produced data with such a high proportion of noise that the scaling models fit very poorly. Finally, the S-D judgment data has the further drawback of not permitting the analysis of individual differences. In order to compute the similarities from this data it was necessary to assume that there were no individual differences.

We conclude, therefore, that the same-different paradigm does give us two additional ways of gathering data useful for psychophysical scaling, although neither way is as desirable as the standard similarity estimation paradigm.

\section{REFERENCES}

BEHRMAN, B. W., \& BROWN, D. R. Multidimensional scaling of visual form: A psychophysical analysis. Perception \& Psychophysics, 1968, 4, 19-25.

BROWN, D. R.. \& ANDREWS, M. H. Visual form discrimination: Multidimensional analysis. Perception \& Psychophysics, 1968, 4 , $401-406$.

CLIFF, N. The 'idealized individual' interpretation of individual differences in multidimensional scaling. Psychometrika, $1968,33,225-323$

CLIFF, N., \& YOUNG, F. W. On the relation between unidimensional judgments and multidimensional scaling. Organizational Behavior \& Human Performance, 1968,3, 269-285.

Fig. 10. Relation between psychophysical functions derived from main subgroup similarity judgments and latency of correct same-different judgments. 
JONES, L. V., JOHNSON, E. S., \& YOUNG, F. W. Computer control of psychologica experiments. In B. Weiss (Ed.), The digital computer in the behavior laboratory. New York: Appleton-Century-Crofts, in press.

KRUSKAL, J. B. Nonmetric multidimensional scaling: A numerical method. Psychometrika, 1964, 29, 115-129.

MARKLEY, R. P., AYERS, D., \& RULE, S.. Similarity judgments of line length. Perception $\&$ Psychophysics, 1969, 6, 58-60.

ROSS, J. A remark on Tucker \& Messick's 'points of view' analysis. Psychometrika, 1966, 31, 27-31.

ROSS, R. T. Optimum orders for the presentation of pairs in the method of paired comparison. Journal of Educational Psychology, 1938, 25, 375-382.
SHEPARD, R. N. The analysis of proximities: Multidimensional scaling with an unknown distance function, I. Psychometrika, 1962, 27, 125-139.

STENSON, H. H., \& KNOLL, R. L. Goodness of fit for random rankings in Kruskal's nonmetric scaling procedure. Psychological Bulletin, $1969,71,122-126$.

TORGERSON, W. S. Theory and methods of scaling. New York: Wiley, 1960.

TUCKER, L. R., \& MESSICK, S. An individual differences model for multidimensional scaling. Psychometrika, 1963, 28, 333-367.

YOUNG, F. W. Nonmetric multidimensional scaling: Recovery of metric information. Psychometrika, in press.

YOUNG, $F$. W., \& PENNELL, R. J. An IBM System $/ 360$ program for points of view analy sis. Behavioral Science, 1967, 12, 166.
YOUNG, F. W., \& TORGERSON, W. S. TORSCA, a FORTRAN IV program for Shepard-Kruskal multidimensional scaling analy sis. Behavioral Science, 1967, 12, 498.

\section{NOTES}

1. This report was supported in part by a PHS research grant, No. M-10006, from the National Institute of Mental Health, Public Health Service, and in part by a Science Development grant, No. GU-2059, from the National Science Foundation.

2. Address: The L. L. Thurstone Psychometric Laboratory, The University of North Carolina, Chapel Hill, North Carolina 27514.

(Accepted for publication March 2, I970.) 